\title{
Context-Aware Mobile Applications: Taxonomy of factors for building approaches
}

\author{
Estevan R. Gómez-Torres \\ Facultad de Ingeniería: Carrera de \\ Ingeniería en Informática, UTE \\ Quito, Ecuador \\ estevan.gomez@ute.edu.ec
}

\author{
Cecilia Challiol \\ UNLP, Facultad de Informática, LIFIA \\ and also CONICET \\ La Plata, Buenos Aires, Argentina \\ ceciliac@lifia.info.unlp.edu.ar
}

\author{
Silvia E. Gordillo \\ UNLP, Facultad de Informática, LIFIA \\ and also CICPBA \\ La Plata, Buenos Aires, Argentina \\ gordillo@lifia.info.unlp.edu.ar
}

\begin{abstract}
Fusion of sensing mechanisms inside mobile devices (e.g.: GPS, accelerometers) have driven the growth of context-aware mobile applications. Currently, there are building approaches for this kind of applications, but these do not have the flexibility, for example, to derive applications combining different location sensing mechanisms. In this paper, we present a first proposal of a taxonomy of factors that could be considered by context-aware mobile application building approaches, in order to provide variability in the kinds of derived applications. The aim is to generate a discussion that can contribute to the unification of aspects that should be addressed by these building approaches. To complement the taxonomy, we present the analysis of an interview that was conducted with regard to developers who use (or could use) these building approaches. We hope this will enrich the discussion in relation to this kind of approaches.
\end{abstract}

Keywords-Context-aware mobile applications, Taxonomy, Sensing mechanisms, Building approaches, Mobile Computing

\section{INTRODUCTION}

In recent years, technological advances have allowed to emergence of a varied range of mobile applications. The incorporation of sensors in mobile devices (for example: GPS, accelerometers) have driven the growth of contextaware mobile applications [1]. While these applications have been studied for several years [2], [3], only in the last years have technological advances enabled these applications to become useful for people (for example, applications that use GPS to provide information or services).

The concept of context has not only been explored by different areas of Computer Science [4] (e.g.: Artificial Intelligence, Home Automation, etc.), but it has also been addressed by different authors from different perspectives [1]. Some of these perspectives are specifically oriented, for example, offering modeling solutions, while others are building approaches for these kinds of applications [1]. That is, context-aware mobile applications are being studied at different levels of abstraction. However, there is still no consensus as to how to classify the contexts [5] or what characteristics should be considered relevant in their development [6]. Therefore, there is currently no unified solution for such applications as mentioned in [1] and [6]. This aspect is currently an open area of research. Moreover, an important issue is how usable these applications are [7].

Development of context-aware mobile applications is a complex task [6], so, developers look for building approaches to speed up this task. There are some approaches aimed at non-expert users [8] and others that require more technical knowledge [9] such as modeling features. These approaches address certain specific aspects of context-aware mobile applications, for example, only GPS is provided as a sensing mechanism. So, the kind of application that could be derived is limited. This restriction is generally related to the available APIs that these approaches use.

Even though, indoor location systems have been explored in the last years [10], [11], [12], there is not yet overall unified solution for these systems as GPS for outdoor spaces. Therefore, this is a limitation to provide these kinds of sensing mechanisms as part of building approaches.

Building approaches speed up creation but they usually lose sight of the concepts already studied with regard to these applications [6]. From this arises the motivation of this paper, to be able to contribute to the unification of factors related to context, in particular, those that should be considered by building approaches so that they are more flexible when generating applications of this kind.

The goal of the paper is to present a first proposal of a taxonomy of factors that could be considered by contextaware mobile application building approaches. The aim is to generate a discussion that can contribute to the unification of factors that should be addressed by these building approaches.

The taxonomy of factors proposed is oriented to provide, in terms of the approaches perspective, varied range of variability [13] in the kinds of generated applications. For example, ones that could be use different kinds of location sensing. For this, a bibliographic analysis has been carried out to identify those factors that are relevant in relation to giving flexibility to building approaches.

To complement the taxonomy, we present the analysis of an interview that was conducted with regard to developers who use (or could use) these building approaches. We hope this will enrich the discussion in relation to these approaches.

This paper is structured as follows. Section 2 describes related work. In Section 3, the taxonomy of factors is presented. The interview carried out in relation to building approaches is analyzed in Section 4. Section 5 generates a discussion space in relation to the theme. The conclusions and future work are described in Section 6.

\section{RELATED WORKS}

The topic of context has been studied for several years now, as is mentioned in [2] and [3]. Each of the authors who has approached it has done so from a specific perspective, or has focused on specific characteristics [1]. Several proposals of frameworks or platforms for context-aware mobile applications are described in [2], [3] and [7]. In this section, the related works are considered it terms of the goal of the presented paper. 
In [6] it is emphasized that, in relation to the context, it is required to consider the level of relevance thereof, the way these are combined, as well as precision and accuracy in relation to the sensed value. For example, precision and accuracy of the GPS, in order to determine how to interpret the sensed value. Moreover, each indoor location sensing mechanisms have its own accuracy [10], [11], [12]. So, according to this, they are more appropriate to a specific kind of application, for example, BLE (Bluetooth Low Energy) is better for indoor navigation and tracking [11]. Therefore, this information to be considered must be configured in some way; however, this is not generally contemplated by building approaches [14].

There is not yet a unified consensus with regard to the categorization of contexts. In [5], 36 context models are analyzed, and five classifications shared by these models are identified: social context, location, time, physical context and user context. On the other hand, the authors analyzed contexts features that are considered by context-aware mobile applications, and it identified 10,498 context elements. When the authors of [5] have tried to match these identified context elements with categorization mentioned, they indicated that more than half did not correspond to any categorization. In this way, it could be appreciated that there is a gap between applications and approaches (in this case, modeling approaches).

In [1] and [7], the authors specify that the contexts can have a passive configuration, which the user performs manually, or an active configuration in which the application is self-configuring based on user behavior (this requires a continuous monitoring and learning mechanism). In addition, it is mentioned that sensing mechanisms can be passive, that is, it requires the intervention of the user (for example, reading QR Codes), or the execution can be active, that is, applications contains an automatic sensing mechanism (for example, GPS). Both configuration and execution are of vital importance in the case of building approaches, where it would be ideal for users (of these approaches) to determine whether they are active or passive. Moreover, some indoor location sensing mechanisms require additional infrastructure, for example, a signals' database to work properly [10].

There are currently a number of building approaches for context-aware mobile applications. For example, the App Inventor [8] is an "online" program, which allows users to create Android applications without having any technical knowledge. The generated applications can include only GPS as a location sensing mechanism. That is, users can only create applications for outdoor spaces. The App Inventor allows configuring precision and accuracy of the location sensor (in this case, GPS). However, it is not possible to combine accelerometer sensor to orientation sensor, in that the configuration reacts to these three sensors separately. In this case, the App Inventor only focuses on some contexts of the device and user's location.

On the other hand, WebRatio Mobile [9] allows users to create context-aware mobile web applications. WebRatio Mobile is oriented to expert users who should have knowledge of databases and hypermedia design. The generated applications are packaged in PhoneGap, allowing to be used on both Android and iOS platforms. The users could define contexts related to Device, Network Connectivity and Position. In this case, location setting is also limited to GPS, allowing only generating applications for outdoor spaces. It can define precision and accuracy related to GPS.

It can be observed that these approaches ([8] and [9]) are limited, in particular focus only of building applications for outdoor spaces, where certain contexts are also considered, most of them related to the available APIs. Moreover, neither [8] nor [9] address the categorization of contexts identified by [5]. For example, they do not consider any aspects with regard to social context or time.

For indoor spaces, there is wide variety of indoor location sensing mechanisms [10], [11], [12]. However, there is not currently overall unified solution for these sensing mechanisms (as it is the case of GPS for outdoor spaces). So, this impacts in order to provide building approaches for indoor applications.

Consequently, it is expected that this paper will be able to contribute to the future discussion; with regard to having more flexible building approaches, for example, generate context-aware mobile applications combined different location sensing mechanisms.

\section{TAXONOMY OF FACTORS PROPOSED}

This section presents a first proposal of a taxonomy of factors that should be considered by context-aware mobile application building approaches. With this taxonomy, it is expected that the building approaches will have varied range of variability [13] in the kinds of generated applications. To determine these factors, a bibliographic analysis was carried out, highlighting especially the following works detailed in Section 2: [1], [5], [6] and [7]; these works were used as references for the specification of the taxonomy proposed.

Figure 1 shows the taxonomy of factors proposed for this paper, with six factors being identified: relevance, combination, precision and accuracy, categorization, type of configuration and type of execution. Below each factor identified in Figure 1 are detailed:

- Relevance: approaches should be able to indicate the relevance of each of the defined contexts. This would allow, for example, generating reduced versions of applications with the most relevant contexts. For now, approaches such as [8] and [9] do not indicate

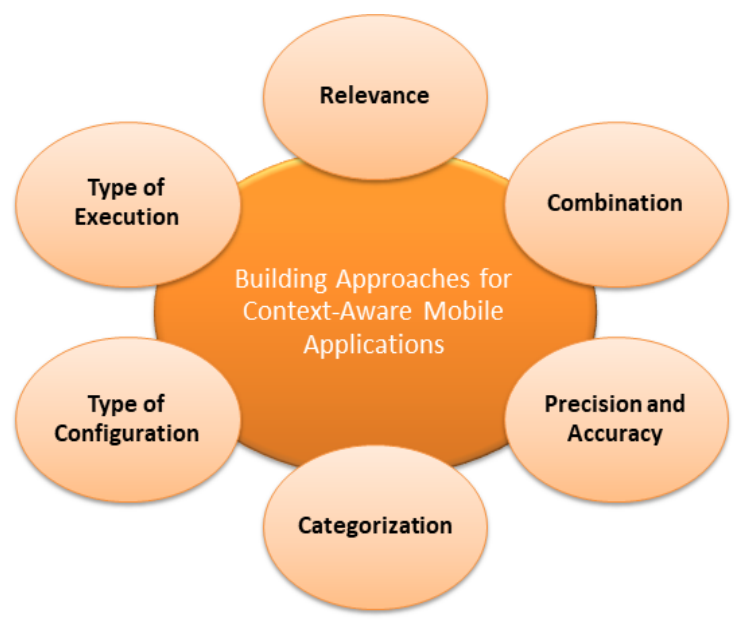

Fig 1. Taxonomy of factors proposed. 
relevance explicitly, but everything defined is relevant.

- Combination: approaches should allow combining contexts. This implies that not only identifying which contexts are combined, but also how they behave when they are considered together. For example, some indoor location sensing mechanisms could be combined in order to work more precisely [10].

- Precision and Accuracy: approaches should specify precision and accuracy for each physical sensor (e.g. GPS), as it is the case in [8] and [9]. Each indoor location sensing mechanisms have its own configuration [10], [11].

- Categorization: approaches should have a way to categorize contexts. For example, contexts of users, environment or mobile objects require to be handled in a completely different way (using different sensing mechanisms), so, categorization will allow to improve how each of them is derived inside applications.

- Type of configuration: approaches should allow the identification of what type the configuration will be, for example, passive or active, as detailed in [1]. According to the type of configuration, approaches should allow to provide more details settings. For example, for a passive configuration it should be possible to define how users (of the generated application) will be able to make this configuration, suppose it is defined a data form with the possible options to set.

- Type of execution: approaches should allow identification of the type of execution associated with each context, for example, passive or active, as detailed in [1]. For each of this possible types, approaches should bring support to set it. For example, in the case of location passive execution (that is, users intervening in some way), approaches should allow to specify how this is addressed. Let's suppose that user has to read a QR code to determine his/her location, it should be indicated (from the approach perspective) how the generated application behaves, for example, when QR code read is incorrect.

Thus, a taxonomy of factors proposed is detailed. Note that this is a first proposal and in the future, we should explore the feasibility of addressing these factors specifically by building approaches for context-aware mobile applications.

Up to now, these factors have been identified to allow them to be addressed by the building approaches, in order to have greater variability [13] in terms of the derived applications. In particular, for indoor location sensing mechanisms factors should be analyzed in more detail to provide more guideline in order to help building approaches, because each mechanisms have its own accuracy or they could be combined better with some others mechanisms [10].

\section{DEVELOPMENT PERSPECTIVE ANALYZED}

This section presents the analysis of an interview that was done regarding to developers who use (or could use) these building approaches. The goal of this interview was to provide a discussion with information complementary to the taxonomy presented in this paper. That is, a building approach should not only address factors of the taxonomy proposed, but also requirements of the developers who use these approaches [7].

The interview involved six university professors with experience in the area of mobile application development. In addition, all the interviewees had taken the course "Mobile Computing" given at the Faculty of Informatics of the National University of La Plata (Argentina) in 2014. In this course, advanced concepts were offered in relation to the design of context-aware mobile applications. That is, the interviewees had basic knowledge in relation to the context concept.

Below are listed the two most relevant questions that were presented to the interviewees, and that are related to this paper:

- Question 1: What building approaches do you know for the development of context-aware mobile applications?

- Question 2: What do you expect from a contextaware mobile application building approach?

Question 1 was open; the interviewees could answer with the name of any approach they knew. The result of this question can be observed in Figure 2 where two approaches known to some interviewees are App Inventor [8] and WebRatio [9] (both described briefly in Section 2). Note that some of the interviewees were not aware of any approach.

In the case of Question 2, it was closed. The interviewees could opt one of the following two options in a nonexclusive way: "generation of applications, without knowledge of specific platforms" or "generate applications that encode low-level code for each specific platform". Figure 3 shows the results for this question. It can be appreciated that $62.7 \%$ preferred to generate applications without having specific knowledge of the platforms.

Although other questions were asked, they do not provide significant results in terms of this paper. From Question 2, it

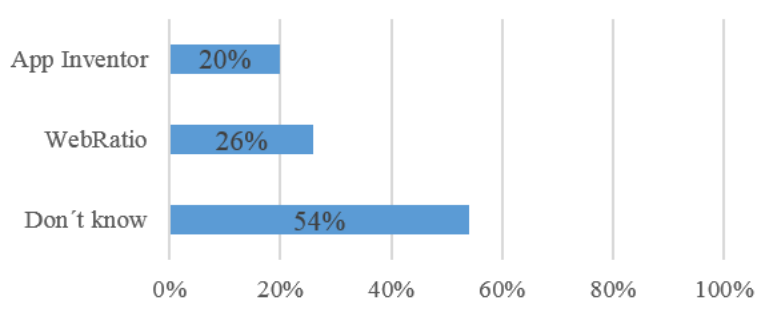

Fig 2. Results of Question 1.

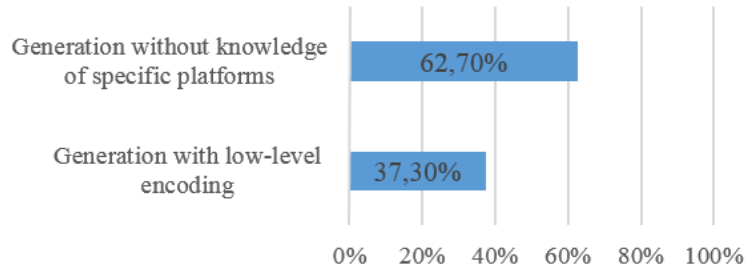

Fig 3. Results of Question 2. 
can be observed that there is a need to have approaches that do not require prior knowledge, but which also offer the possibility to experts of making code configurations at lowlevel. For the latter, a building approach could use the taxonomy of factors proposed to achieve greater flexibility in the generated applications. These was an initial interviews, we know that with some few people and two questions could not be extract any significant conclusion. So, other user's profiles should be interviewed (e.g. teachers [15]), in order to understand what a building approach requires for each specific domain.

\section{DISCUSSION}

In this section, a discussion is presented in relation to the theme of building approaches for context-aware mobile applications, and what it is proposed in this paper.

The factors of relevance, combination, precision and accuracy are oriented towards building approaches for expert users, for example as detailed in [9]. This is because a nonexpert user may be unaware of the meaning of, for example, accuracy. In the case of the categorization of context depending on how it is presented from the approach could be understood by non-expert users. For example, if it is the context of the user or the environment in which they are moving.

Existing approaches (whether oriented to expert users or not, for example [8] and [9]), generally generate applications for outdoor spaces, due to having access to GPS APIs. With advancements of new mechanisms relating to indoor location ([10], [11] and [12]), it will be expected that, in a near future, the range of applications that could be generated be expanded. For this, it is going to be essential to be able to incorporate the factors of the taxonomy proposed in this paper.

What users/developers will be able to generate depend on the flexibility provided by building approaches. That is why we believe that the taxonomy of factors proposed could contribute to improving the flexibility of approaches. This in turn will contribute to the expectations of users with regard to such approaches, as is mentioned in Section 4.

\section{CONCLUSION AND FUTURE WORK}

In this paper, it is presented a first proposal of a taxonomy of the factors that could be considered by building approaches for context-aware mobile applications, in particular, when it comes to achieving greater variability in the kinds of generated applications. It is expected that this paper will contribute to the discussion in relation to this topic; especially how to move towards a unified solution that can to address building context-aware mobile applications.

An interview was also presented reflecting the expectations of expert users in relation to building approaches. Some of them expect that building approaches allow setting low-level details in order to have more control over the generated applications. In this case, approaches would require to be enriched with more low-level setting according to, for example, how each platform manages every sensing mechanism.
We are working on defining a building approach for context-aware mobile applications based on the taxonomy of factors proposed. Allowing in this way for developers not only generate applications for outdoor spaces, but also for indoor spaces. As a result of this, the taxonomy proposed could be enriched with new factors that may arise.

As a future work, we will explore how to enrich our taxonomy with others related existing works that explore complementary aspects to context-aware mobile applications. As for example, in [16] the authors present a taxonomy related to context-aware computing and dynamic software variability management.

\section{REFERENCES}

[1] U. Alegre, J. Augusto, and T. Clark, "Engineering context-aware systems and applications : A survey," Journal of Systems and Software, vol. 117, pp. 55-83, July 2016.

[2] A. Dey, "Understanding and Using Context," Personal and Ubiquitous Computing, vol. 5, no 1, pp. 4-7, Feb. 2001.

[3] B. Schilit, "Context-Aware Computing Applications," Proc. of the First Workshop on Mobile Computing (WMCSA 1994.), IEEE, Dec. 1994, pp. 85-90.

[4] J. Augusto, A. Aztiria, D. Kramer, and U. Alegre, "A Survey on the Evolution of the Notion of Context-Awareness," Applied Artificial Intelligence, vol. 31, no. 7-8, pp. 613-642, Feb. 2018

[5] C. Bauer and A. Novotny, "A consolidated view of context for intelligent system," Journal of Ambient Intelligence and Smart Environments, vol. 9, no 4, pp. 377-393, Jun. 2017.

[6] C. Bauer and A.K Dey, "Considering context in the design of intelligent systems: Current practices and suggestions for improvement," Systems and Software, vol. 112, pp. 26-47, Feb. 2016.

[7] U. Alegre-Ibarra, J.C. Augusto, and E. Carl, "Perspectives on engineering more usable context aware systems," Journal of Ambient Intelligence and Humanized Computing, pp. 1-17, May 2018.

[8] D. Wolber, "App Inventor and Real-World Motivation," Proc. of the 42nd ACM technical symposium on Computer science education, ACM, March. 2011, pp. 601-606.

[9] M. Brambilla and P. Fraternalli, "Interaction flow modeling language: Model-driven UI engineering of web and mobile apps with IFML," Morgan Kaufmann, 2015.

[10] P. Davidson, and R. Piché, "A survey of selected indoor positioning methods for smartphones," IEEE Communications Surveys \& Tutorials, vol. 9, no. 2, pp. 1347-1370, 2017.

[11] A. Basiri, E.S. Lohan, T. Moore, A. Winstanley, P. Peltola, C. Hill, and P.F. Silva, "Indoor Location Based Services Challenges, Requirements and Usability of Current Solutions," Computer Science Review, vol. 24 , pp. 1-12, May 2017.

[12] R.F. Brena, J.P García-Vázquez, C.E Galván-Tejada, D. MuñozRodriguez, C. Vargas-Rosales, and J. Fangmeyer, "Evolution of Indoor Positioning Technologies: A Survey," Journal of Sensors, vol. 2017, article ID 2630413, Mar. 2017.

[13] A. Fortier, G. Rossi, S.E. Gordillo, and C. Challiol, "Dealing with variability in context-aware mobile software," Journal of Systems and Software, vol. 83, no. 6, Jun. 2010, pp. 915-936.

[14] M. Nieke, G. Engel, and C. Seidl, "DarwinSPL: an integrated tool suite for modeling evolving context-aware software product lines," Proc. of the Eleventh International Workshop on Variability Modelling of Software-intensive Systems, ACM, Feb. 2017, pp. 9299.

[15] J. Joo-Nagata, F.M. Abad, J.G.B Giner, AND F.J García-Peñalvo, "Augmented reality and pedestrian navigation through its implementation in m-learning and e-learning: Evaluation of an educational program in Chile", Computers \& Education, vol. 111, pp. 1-17, Aug. 2017.

[16] K., Mens, R. Capilla, N. Cardozo, and B. Dumas, "A taxonomy of context-aware software variability approaches," Proc. of the 15th International Conference on Modularity, ACM, Mar. 2016, pp. 119124 